\title{
Editorial
}

\section{Angionegative Subarachnoid Hemorrhage: Need for Consensus Guidelines}

\author{
Girish Menon ${ }^{1, \odot}$ \\ ${ }^{1}$ Department of Neurosurgery, Kasturba Medical College, Manipal, \\ India
}

J Neurosci Rural Pract 2020;11:517-518

In approximately 10 to $20 \%$ cases of spontaneous nontraumatic subarachnoid hemorrhage (SAH), no underlying vascular etiology is found and the source of hemorrhage remains unknown. ${ }^{1,2}$ This angionegativity has been ascribed to various factors including venous bleeds, hypertension, alcoholism, and drug abuse. However, these need to be a diagnosis of exclusion and to be considered only after ensuring the absence of an aneurysm. If an aneurysm is missed in the first angiogram, these patients are at risk for aneurysmrelated complications. Definition of angionegativity, yield of repeat angiography, protocol for angiographic follow-up, etc. are all controversial topics with no well-defined guidelines. The authors of this manuscript share their experience with 119 angionegative SAH patients and discuss the dilemmas in management of such patients. ${ }^{1}$ Their concluding suggestion to perform a repeat digital subtraction angiography (DSA) for all patients with nonperimesencephalic (nPM)-SAH at 6 weeks after the initial bleed appears to be the most appropriate protocol with the currently available level of evidence.

Angionegative SAH patients can be (1) computed tomography (CT) negative, SAH confirmed by lumbar puncture; (2) perimesencephalic hemorrhage (PM-SAH); and (3) classic $\mathrm{nPM}-\mathrm{SAH}$. The quantum of bleed is highest in (nPM-SAH) and so is the possibility of an underlying aneurysm. Complications are also proportionately higher in this group emphasizing the importance of ruling out an aneurysm. Although the need for repeat angiographies to avoid missing an aneurysm is justified, the yield is surprisingly poor. In the authors series, of the 98 patients who underwent repeat angiography a vascular abnormality was detected in only two patients. Both the abnormalities were minor irregularities in a vessel which did not require any surgical intervention, and both were noted in patients with nPM-SAH. ${ }^{1}$ This finding is at variance with a recent meta-analysis on the yield of a repeat angiography, which revealed a pick up rate of approximately $10 \%$ in nPM$\mathrm{SAH}$, and $1.6 \%$ for patients with perimesencephalic SAH. ${ }^{3}$
These conflicting reports add to the controversies related to angionegative $\mathrm{SAH}$ which can be summarized as follows:

A. How does one define angionegativeness? What is the gold standard? Is it DSA or computed tomographic angiography?

The diagnostic protocol for management of angionegative SAH varies from institution to institution. Most aneurysm surgeons prefer to perform all three, a computed tomographic angiography (CTA), DSA, and a brain/cervical spine magnetic resonance imaging (MRI), before labeling a case as angionegative SAH. With a pooled sensitivity and specificity of 98 and $100 \%$, CTA has now replaced DSA as the first mode of investigation in almost all centers and DSA is reserved for patients with suspicious CTA findings. In spite of being the gold standard, DSA has the drawback of being operator and interpretation dependent, and aneurysms may be missed even on biplane DSA. ${ }^{4}$ DSA may miss an aneurysm due to copious cisternal blood obscuring the aneurysm, vasospasm, thrombosis, small size of the aneurysm, or simply due to a technically inadequate examination. A complete four vessels cerebral angiogram with appropriate views performed by an experienced and competent surgeon/radiologist is mandatory in all doubtful cases of SAH. The diagnostic yield of cervical spine and brain MRI in angionegative SAH is low and reported to be around 1.3 to $4 \%{ }^{5}$ In the absence of accepted guidelines, it appears prudent to perform all the three modalities on admission.

B. What is the yield of repeat angiography? What is the minimum angiographic follow-up required?

Available data on the yield of repeat angiography is limited, and there is an ongoing debate on the utility of repeated angiographic imaging. ${ }^{4}$ The yield of repeat angiogram in PM-SAH has been uniformly poor. In a 2014 published meta-analysis of 1,031 patients with angionegative 
PM-SAH repeat angiogram detected aneurysms only in eight patients. In another recent meta-analysis of 13 studies with 588 patients, a vascular etiology was detected in only three patients with isolated perimesencephalic SAH. ${ }^{4}$ However, the diagnostic yield of repeat angiography for patients with nPM-SAH is higher and to the tune of $10 \%{ }^{6}$ The diagnostic yields of repeat DSA for nPM-SAH was 7.4\% in another multicentric retrospective study published in 2015 by Sadigh et $\mathrm{al}^{5}$. The protocol adopted by the Barrow Ruptured Aneurysm Trial (BRAT) group protocol which includes a DSA and MRI if CTA is negative followed by a repeat angiography 1 week later is widely followed at many centers including ours. ${ }^{2}$

C. What are the possible complications in patients with angionegative SAH compared with aneurysmal SAH patients?

In general, patients with an SAH of unknown etiology have a benign clinical course. However, delayed cerebral ischemia, hydrocephalus, and rebleeding have been reported. ${ }^{2}$ In the subgroup analysis of angionegative patients in the BRAT, it was observed that none of these patients had a rebleed, but delayed cerebral ischemia (DCI) was observed in $7 \%$, hydrocephalus in $28 \%$, and a need for shunt in $7 \%$. These complications were understandably more in patients with in the classic nPM-SAH where the hemorrhage volumes tend to be greater. Vasospasm and DCI are rare in patients with perimesencephalic hemorrhage, and when present, it tends to resolve without significant neurological deficits. Overall outcome too depends on the quantum of blood and in the BRAT study subgroup only $79 \%$ of patients with nPM-SAH achieved complete independent recovery, as compared with $95 \%$ of patients with PM-SAH. ${ }^{2}$

Summarizing the above observations, it is fair to conclude that prior to confirming a diagnosis of angionegative $\mathrm{SAH}$, a patient should undergo all three CTA, DSA cervical, and brain MRI in that order during the first admission. Since the risk of false negativity and complications are low with PM-SAH and CT negative SAH, these subset of patients may be closely followed up without the need for a subsequent angiogram. Angionegative nPM-SAH patients need a higher level of scrutiny and need to undergo follow-up DSA at a competent center within the first 6 weeks to rule out any possibility of a missed vascular pathology. Long-term angiographic follow-up beyond 6 weeks may not be necessary as delayed rebleeding in such patients is an unlikely event.

\section{Funding}

None.

\section{Conflict of Interest}

None declared.

\section{References}

1 Yeole U, Nagesh M, Shukla D, HR A, AR P. The yield of repeat angiography in angiography-negative spontaneous aubarachnoid hemorrhage. J Neurosci Rural Pract 2020;11(4):565-572

2 Elhadi AM, Zabramski JM, Almefty KK, et al. Spontaneous subarachnoid hemorrhage of unknown origin: hospital course and long-term clinical and angiographic follow-up. J Neurosurg 2015;122(3):663-670

3 Kalra VB, Wu X, Matouk CC, Malhotra A. Use of follow-up imaging in isolated perimesencephalic subarachnoid hemorrhage: a meta-analysis. Stroke 2015;46(2):401-406

4 Geng B, Wu X, Brackett A, Malhotra A. Meta-analysis of recent literature on utility of follow-up imaging in isolated perimesencephalic hemorrhage. Clin Neurol Neurosurg 2019;180: 111-116

5 Sadigh G, Menon RK, Bhojak M, et al. Radiological management of angiographically negative, spontaneous intracranial subarachnoid hemorrhage: a multicenter study of utilization and diagnostic yield. Neurosurgery 2019;85(1):126-133

6 Bakker NA, Groen RJ, Foumani M, et al. Repeat digital subtraction angiography after a negative baseline assessment in nonperimesencephalic subarachnoid hemorrhage: a pooled data meta-analysis. J Neurosurg 2014;120(1):99-103 